\title{
Implicaturas generalizadas en español: un abordaje experimental
}

Generalized Implicatures in Spanish: An experimental approach

\begin{abstract}
Rodrigo Loredo
Universidad de Buenos Aires, Facultad de Filosofía y Letras, Instituto de Lingüística

Juan Kamienkowski

Universidad de Buenos Aires, Facultad de Ciencias Exactas y Naturales, Laboratorio de Inteligencia Artificial Aplicada

Virginia Jaichenco Universidad de Buenos Aires, Facultad de Filosofía y Letras, Instituto de Lingüística
\end{abstract}

\section{Resumen}

Las implicaturas conversacionales generalizadas (ICG) son un tipo de inferencia pragmática que se caracteriza por una derivación que sigue ciertas regularidades y que es relativamente independiente del contexto de situación (Grice, 1989). Existen dos modelos de procesamiento sobre este fenómeno desde una perspectiva cognitiva (Noveck \& Reboul, 2008): 1) el modelo del procesamiento por default (Levinson, 2000) sostiene que el significado pragmático de las ICG se procesa automáticamente y que el contexto interviene tardíamente; 2) el modelo de procesamiento guiado por el contexto (Sperber \& Wilson, 1995) considera que la información contextual interviene tempranamente para iniciar el proceso de derivación. Diversos estudios experimentales han cuestionado las hipótesis de estos modelos (Bezuidenhout \& Cutting, 2002; Breheny, Katsos \& Williams, 2006), sin embargo, a pesar de utilizar las mismas metodologías arribaron a resultados contrapuestos. En este trabajo reportamos los resultados de un experimento de juicios de aceptabilidad realizado con la implicatura escalar producida por el cuantificador algunos (Horn, 1984). Los resultados sugieren que el contexto que precede a la implicatura influye en su procesamiento tardíamente, en línea con las predicciones del modelo por default. 
Palabras clave: cuantificador existencial; implicaturas escalares; juicios de aceptabilidad; tiempos de lectura; pragmática experimental

\section{Abstract}

Generalized conversational implicatures (GCIs) are a type of pragmatic inference characterized by a derivation following certain regularities and is relative independent from the context (Grice, 1989). There are two processing models on this phenomenon, from a cognitive perspective (Noveck \& Reboul, 2008): 1) the default processing model (Levinson, 2000) states that the pragmatic meaning of GCIs is processed automatically and that contextual assumptions are used in later stages; 2) the guided-by-context model (Sperber \& Wilson, 1995) stems from that contextual assumptions are integrated in early stages to prompt the derivation process. Several experimental studies have tested the hypotheses of these models (Bezuidenhout \& Cutting, 2002; Breheny, Katsos \& Williams, 2006). Nevertheless, they have arrived to opposite results using the same methodologies. This paper reports the results of an acceptability judgment experiment that uses the scalar implicature triggered by the existential quantifier algunos (closely related to some in English) (Horn, 1984). The results suggest that the context that precedes the implicature is integrated to the derivation process in a later stage, in line with the predictions of the default model.

Keywords: existential quantifier; scalar implicatures; acceptability judgments; reading times; experimental pragmatics 


\section{Introducción}

Las implicaturas conversacionales son propuestas por Grice (1989: 22-41) para explicar la diferencia que, a menudo, se encuentra entre el significado de una oración codificado por la sintaxis y la semántica (lo dicho) y el significado pragmático derivado en una situación comunicativa determinada (lo comunicado). La distinción entre estos dos significados obliga a Grice a plantear un mecanismo que permite, a partir de un principio de cooperación (PC) y de máximas, derivar inferencialmente el significado de una oración en su contexto. Sin embargo, Grice señala que hay casos donde la derivación presenta ciertas regularidades, en especial cuando se trata de las implicaturas que dependen de la máxima de cantidad, que se diferencian de otros casos donde la derivación es más dependiente del contexto y no presenta regularidades. Por lo tanto, propone una distinción entre dos tipos de implicaturas que se basa en el rol que ocupa el contexto en la interpretación y la existencia o no de una interpretación preferida por defecto, dos hechos que influyen en los mecanismos de procesamiento de las mismas.

\subsection{Dos tipos de implicaturas}

El primer tipo de implicatura propuesta por Grice es el de implicatura generalizada (IG). Las IG ocurren con un ítem léxico o una estructura sintáctica que genera, de manera relativamente independiente del contexto de situación, una interpretación pragmática que difiere de la que está codificada lógicamente. Un caso paradigmático de IG son las llamadas implicaturas escalares (Horn, 1984), en las que el uso de un ítem de una escala implica la negación de los ítems que se encuentran en los niveles siguientes por observación de la máxima de cantidad (Grice, 1989: 26). Por ejemplo, en (1), para la escala \{algunos, todos $\}$ el uso de algunos $x$ implica pragmáticamente la negación de todos los $x$ (algunos y no todos), a pesar de que lógicamente esta implicación no está codificada en la forma lógica, ya que el cuantificador existencial también es verdadero para todos los x (algunos y posiblemente todos) (cfr., Levinson, 1983: 134, para otras escalas). 
(1) ¿Aprobaste los parciales?

Aprobé algunos.

Sin embargo, este tipo de escalas no se pueden considerar derivaciones convencionales codificadas léxicamente, ya que hay casos en los que el uso de algunos funciona con su significado lógico y no implica la negación de todos, como se puede ver en (2).

(2) Aprobé algunos, de los otros no sé la nota, pero probablemente también los aprobé.

Así, este tipo de implicatura se apoya en la ambigüedad que existe entre el significado dado por la forma lógica y el que se alcanza por la derivación de la inferencia. Esto ocurre en mayor medida con operadores lógicos como los cuantificadores, la conjunción y la disyunción. De la misma forma, también pueden considerarse implicaturas generalizadas los pedidos indirectos o actos de habla indirectos (Searle, 1975), donde la forma lógica de la oración codifica el modo interrogativo mientras que su interpretación implicada es la de una pregunta y un pedido. Sobre este punto, Levinson $(1983,2000)$ ha señalado que las IG ocupan un lugar especial dentro del procesamiento inferencial, ya que parecerían estar sujetas a un mecanismo que, por defecto, toma la interpretación pragmática como preferida en cualquier contexto de situación, mientras que la interpretación lógico-semántica solo se habilita cuando se produce una situación especial que cancela la inferencia.

El otro tipo de implicatura se denomina particularizada (IP), ya que su interpretación está sujeta a un contexto de situación particular. Es decir, no depende de un ítem léxico específico o una estructura sintáctica particular, sino que requiere de un proceso metarrepresentacional, donde el contexto cumple un rol más importante y la derivación del significado implicado se apoya fuertemente en el mantenimiento del PC y, por lo general, en la observación de las máximas de modo o de relevancia (Grice, 1989: 37). En (3), por ejemplo, es necesario apelar al contexto para derivar el significado de lo dicho por Sara.

\section{(3) Ana: ¿Estás bien, Sara? \\ Sara: Tengo mucho frío.}


En este caso, tengo mucho frío puede ser interpretado de diversas maneras (Sara está enferma, Sara quiere que Ana prenda la calefacción, Sara quiere que Ana apague el aire acondicionado, etc.), por consiguiente, la lectura relevante se derivará a partir de las circunstancias del contexto particular que enmarca la conversación.

De esta manera, las diferencias entre ambos tipos de implicaturas están centradas en el rol que ocupa el contexto en el proceso derivativo y en la posibilidad de un mecanismo por default que procese las implicaturas generalizadas sin recurrir a la integración temprana del contexto en la derivación. En ambos casos, se asume que el principio de cooperación se mantiene y que las implicaturas surgen por la observación o violación de alguna de las máximas.

Finalmente, también cabe mencionar la distinción entre implicaturas conversacionales y convencionales. Esta diferencia se indica para referir, en este último caso, a implicaturas que surgen de construcciones léxicas o sintácticas que dan lugar a significados no codificados formal o semánticamente, pero en las que el contexto no influye en ningún grado. De la misma manera, las implicaturas convencionales no son explicadas apelando al PC o a las máximas sino a las convenciones lingüísticas de uso de esas construcciones. Por ejemplo, el uso de pero en construcciones adversativas da lugar a una oposición que no se encuentra codificada lógicamente pero que siempre se deriva, independientemente del contexto en el que aparece. Desde el punto de vista formal-lógico, pero es una conjunción y al invertir los términos se mantienen los valores de verdad, aunque la implicación es diferente. Las implicaturas convencionales se distinguen de las generalizadas en cuanto a que el uso de pero siempre da lugar a ese significado de oposición, independientemente del contexto, mientras que, en el caso de las generalizadas, se produce la interpretación implicada en la mayoría de los casos, pero es posible que ciertos contextos lo anulen.

\subsection{Dos modelos para el procesamiento de implicaturas generalizadas}

El primer trabajo que aborda de manera experimental el procesamiento de las implicaturas generalizadas propone dos modelos de procesamiento (Bezuidenhout \& Cutting, 2002). 
Por un lado, el modelo literal-first serial (LFS) propone que inicialmente se recupera la forma lógica de la oración (algunos y posiblemente todos) y luego, mediante un razonamiento no deductivo basado en la información contextual, se enriquece para llegar a la interpretación implicada (algunos y no todos). Para este modelo, el contexto, entendido como un conjunto de representaciones mentales sobre la situación y el conocimiento del mundo compartido, interviene tempranamente luego de que se ha recuperado la forma lógica (Sperber \& Wilson, 1995). Debido a que la interpretación implicada se genera solo si la información contextual la habilita, no existe la cancelación a posteriori de una implicatura, sino que la implicatura nunca se genera si el contexto no la favorece. En este sentido, el modelo LFS no permite realizar una distinción entre implicaturas generalizadas o particularizadas, ya que el proceso inferencial es el mismo para estos diferentes tipos de implicaturas. La forma lógica o significado literal es el input de la derivación y el contexto interviene tempranamente para guiar el enriquecimiento de esta forma y arribar al significado implicado.

El otro modelo propuesto, denominado ranked-parallel o de procesamiento por default, sostiene que en el caso de las implicaturas generalizadas la interpretación literal y la implicada se procesan simultáneamente. El significado implicado se genera automáticamente (Levinson, 2000) y compite por la activación con el significado literal en un proceso en paralelo. Para esta propuesta, las características empíricas observadas en las implicaturas generalizadas se deben a que el significado implicado posee un umbral de activación menor a la del significado literal. De esta manera, el contexto interviene tardíamente una vez que ambas representaciones se han generado para cancelar o inhibir la implicatura y favorecer la interpretación literal. Esto supone que en los casos donde las implicaturas generalizadas deben interpretarse literalmente haya un mayor costo cognitivo ligado al proceso de inhibición o de cancelación de la implicatura generada.

\subsection{Antecedentes experimentales}

Con el objetivo de poner a prueba estos dos modelos, se ha llevado a cabo una serie de estudios que, lejos de ofrecer evidencia definitiva, han mostrado que es necesario sumar nuevos datos y mejorar los diseños experimentales utilizados (Katsos, 2009). Los trabajos que abordaron esta temática consistieron en reali- 
zar comparaciones entre el procesamiento de frases con implicaturas que eran precedidas por diferentes contextos de situación, los cuales podían favorecer la interpretación de la implicatura o funcionar como contextos neutros donde la interpretación literal era la preferida. Estos estudios se realizaron en inglés, francés y griego y se utilizaron principalmente experimentos de tiempos de lectura a ritmo personal, aunque en algunos casos también se efectuaron mediciones de potenciales evocados y seguimiento de movimientos oculares. En esta serie de estudios hubo dos tipos de resultados. Por un lado, los trabajos de Bezuidenhout y Cutting (2002), Lauter (2013), Lewis (2013), Politzer Ahles, Fiorentino, Jiang y Zhou (2013), Politzer Ahles y Husband (2018) muestran datos en concordancia con el modelo de procesamiento por default, ya que no encuentran diferencias significativas entre el procesamiento en los casos donde el contexto precedente no favorece la implicatura, o bien encuentran un mayor costo de procesamiento evidenciado en mayores tiempos de lectura o mayor amplitud en componentes de potenciales evocados como el N400. Por otro lado, Bergen y Gordner (2012), Breheny et al. (2006), De Neys y Schaeken (2007) y Huang y Snedeker (2009) reportan resultados contrapuestos que apoyan el modelo LFS. En este sentido, estos autores presentan datos donde el tiempo de lectura para los estímulos con contextos neutros es menor, mientras que en los casos con contextos que facilitan la implicatura es mayor el rango de tiempo. La variabilidad en resultados muestra lo complejo del fenómeno y da cuenta de la necesidad de nueva evidencia experimental.

En este trabajo reportamos los resultados de un experimento en español, con estímulos que utilizan una estructura dialogal, que busca analizar la relación entre contextos y frases con cuantificadores y dar cuenta de la influencia del contexto en el procesamiento de implicaturas. Tres aspectos diferencian este estudio de los realizados hasta el momento. En primer lugar, los experimentos previos utilizan estímulos con estilo indirecto u oraciones declarativas y condicionales, mientras que en nuestro trabajo usamos diálogos que recuperan las características conversacionales de las implicaturas. En segundo lugar, el uso de juicios de aceptabilidad es una metodología que no se había empleado para analizar la relación entre los contextos y las frases con implicaturas. Finalmente, la falta de trabajos en español sobre implicaturas permite abordar el fenómeno 
desde una lengua con características diferentes a las utilizadas en experimentos previos y proveer nuevos datos para el análisis.

\subsection{Juicios de aceptabilidad}

La elección de un juicio de aceptabilidad con tiempos de reacción para este trabajo surge de dos motivaciones. Por un lado, los trabajos experimentales realizados hasta el momento sobre cuantificadores han utilizado solamente juicios de aceptabilidad como una tarea secundaria con la finalidad de confirmar la naturalidad pragmática de los estímulos. En este sentido, el diseño de estos juicios no incluía hipótesis sobre la interacción entre el contexto y el cuantificador en la evaluación de los estímulos, algo que podría brindar datos complementarios a los obtenidos con otras metodologías. En nuestro caso, el diseño nos permite comparar estas interacciones y brindar evidencia adicional sobre el fenómeno.

Por otro lado, pero relacionado con lo anterior, consideramos que los juicios de aceptabilidad son una herramienta muy útil en los estudios de procesos pragmáticos dada la naturaleza dual que estos presentan. Es decir, hay aspectos del procesamiento pragmático que se consideran automáticos y dependientes de un módulo, en el sentido fodoriano, especializado en operaciones pragmáticas (Sperber \& Wilson, 2002), aunque también existen factores que afectan el procesamiento pragmático que no se procesan de manera modular - como la cortesía o las normas socioculturales de interacción-, pero que pueden intervenir en los resultados de los experimentos que buscan caracterizar los procesos modulares. Por este motivo, es importante tener mayores precauciones a la hora de utilizar variables como el contexto de situación, ya que la naturaleza de esta variabilidad puede deberse a múltiples factores. Así, al utilizar un juicio de aceptabilidad, podemos verificar si las variables independientes manipuladas (contexto neutro vs. contexto facilitador) varían efectivamente en función de la facilitación o no de la implicatura y si no hay otros factores que puedan estar interviniendo en el procesamiento, en este caso, del cuantificador algunos.

Finalmente, es necesario hacer un comentario adicional sobre la interpretación de los resultados en los juicios de aceptabilidad con tiempos de reacción. Tradicionalmente, se relaciona el grado de aceptabilidad con el costo de procesamiento del estímulo a evaluar (Topolinski \& Strack, 2009). De la misma ma- 
nera, cuando se toman en cuenta los tiempos de reacción en tareas, por ejemplo, de decisión léxica o de lectura, la latencia también se relaciona con el costo de procesamiento de los estímulos. Sin embargo, existe poco consenso sobre cómo analizar la relación entre el grado de aceptabilidad y el tiempo que lleva realizar el juicio, en especial cuando estos difieren. En este último caso, las diferencias se pueden deber a que los juicios de aceptabilidad dependen tanto de conocimiento explícito como de conocimiento implícito (Dienes \& Scott, 2005) y que la evaluación de aceptabilidad refleja en mayor medida el acceso al conocimiento explícito mientras que el tiempo de reacción depende, en cambio, del acceso al conocimiento implícito.

\subsection{Descripción del cuantificador algunos en español y su contraste con some en inglés}

En este trabajo utilizaremos el cuantificador algunos que presenta, en líneas generales, un paralelismo con some, cuantificador del inglés, el más abordado en la literatura sobre implicaturas escalares. Desde el punto de vista gramatical, se utiliza el término cuantificador como una categoría transversal "que expresa cantidad, número o grado" (Asociación de Academias de la Lengua Española y Real Academia Española, 2009: 1377 y ss.) y que incluye palabras que pertenecen a diferentes clases. En nuestro caso particular, el uso de algunos que abordamos aquí se corresponde con su uso adjetival de cuantificación de entidades individuales en plural. En función de su significado, se clasifica como un cuantificador de existencia, no universal, indefinido o débil. Dado que, a diferencia del inglés, algunos posee flexión en género y número, cabe destacar que la implicatura generalizada que se analiza solo se halla presente cuando la entidad cuantificada es plural, mientras que cuando el cuantificador opera sobre entidades singulares o incontables no es posible dar lugar a la implicatura. No obstante, esto también ocurre en inglés, cuando some cuantifica una entidad en singular o entidades incontables. En este caso, alterna con any y funciona de manera similar a algún, alguna y a los artículos indefinidos singulares en español (Huddleston \& Pullum, 2002). Finalmente, también es preciso mencionar que el uso de algunos en español puede alternar con el artículo indefinido plural (unos, unas), contraste inexistente en inglés, donde esta distinción se realiza con la entidad cuantifi- 
cada en plural sin cuantificador (cfr., Juan compró unos libros con Juan bought $\varnothing$ books).

\section{Experimento}

En este experimento los participantes debían evaluar en una escala de Likert de 7 puntos el grado de naturalidad de 20 grupos de diálogos emparejados en cuatro condiciones: a) un contexto facilitador + cuantificador algunos (CFA), b) un contexto facilitador + cuantificador solo algunos (CFS), c) un contexto neutro + algunos (CNA) y d) contexto neutro + solo algunos (CNs). Asimismo, se utilizaron dos tipos de estímulos de control que presentaban oraciones aceptables (EAC) y oraciones inaceptables (EIC).

\subsection{Hipótesis}

Con este experimento nos proponemos evaluar dos aspectos sobre el procesamiento de las implicaturas escalares. En primer lugar, nos interesa comprobar la influencia del contexto sobre la interpretación de la frase con cuantificadores. En este sentido, se espera que en el juicio de aceptabilidad haya una diferencia entre la condición CNS y las otras (CNA, CFA, CFS), ya que en este caso se combina un contexto que no facilita la interpretación pragmática (algunos y no todos) y un operador que hace explícita esta interpretación (solo algunos). Esta incongruencia se debería expresar en los resultados como un menor nivel de aceptabilidad para estos casos. En las otras condiciones no debería haber diferencias significativas en los valores de aceptabilidad, ya que en todos los casos el contexto es compatible con el cuantificador.

En segundo lugar, dado que el tiempo de respuesta de los sujetos puede tomarse como evidencia del modelo de procesamiento que subyace a la interpretación de los cuantificadores, deberíamos encontrar una diferencia entre CNA y CFA (y la falta de diferencia entre CNS y CFS). Un mayor tiempo de respuesta para CNA sugerirá que la interpretación pragmática se genera automáticamente y luego se cancela, lo que repercute en el tiempo de lectura y posterior evaluación del diálogo, tal como propone el modelo por default. En cambio, un mayor 
tiempo de lectura para CFA indicará que el procesamiento del significado pragmático es más costoso, ya que se debe integrar el contexto para arribar a esta interpretación, mientras que en los casos donde la interpretación es la lógica este proceso no sería necesario.

Finalmente, también deberíamos encontrar una diferencia en los estímulos de control. Los casos de EIC deberían ser evaluados con un promedio cercano al 0 (el menor grado de aceptabilidad posible) mientras que los casos de EAC deberían tener una evaluación cercana a los estímulos blanco (entre 4 y 6).

\subsection{Método}

\subsubsection{Participantes}

Los participantes fueron 260 argentinos hablantes nativos de español, de entre 18 y 39 años de edad (143 eran mujeres y 117, hombres). En cuanto a su formación, 139 eran estudiantes universitarios mientras que 121 ya habían finalizado sus estudios universitarios o se encontraban cursando estudios de posgrado. Los participantes fueron contactados mediante redes sociales.

\subsubsection{Materiales}

Se utilizaron cuatro listas de 40 estímulos divididos en seis condiciones. Los estímulos presentaban tres partes: un contexto que introducía una situación, una pregunta relacionada con esa situación y una respuesta a esa pregunta. Como se observa en (4) —se señalan en cursivas los contextos y en redondas los segmentos críticos-, la primera condición (CFA (4a)) consistía en un contexto facilitador de la interpretación pragmática, una frase con el cuantificador algunos y una frase que hace referencia a el resto de los elementos cuantificados. La segunda condición (CNA (4b)), presentaba un contexto neutro y la misma frase con cuantificador de la condición anterior. En este caso, no se utilizaba la frase con el resto, ya que, al ser un contexto neutro, la referencia a un resto no habilitado por el cuantificador haría que la evaluación de los diálogos fuera marcadamente negativa. La tercera y cuarta condiciones (CFS (4c) y CNS (4d)) repetían los contex- 
tos facilitadores y neutros pero utilizaban en la frase final la forma explícita solo algunos. La quinta condición (EAC (4e)) consistía en diálogos que no presentaban estructuras con cuantificadores y que eran considerados, a priori, como aceptables. Finalmente, la sexta condición (EIC (4f)) estaba conformada por diálogos del mismo tipo que EAC pero que se construyeron con la intención de que fueran inaceptables. Estos diálogos poseían incoherencias entre el contexto, la pregunta y la respuesta.

(4) Ejemplos de estímulos por condición

a. Contexto facilitador + cuantificador algunos (CFA)

El viernes envié unos regalos a mi familia para Navidad.

¿Llegaron a tiempo?

Llegaron algunos paquetes. El resto no llegó.

b. Contexto neutro + algunos (CNA)

El jueves en la oficina Pedro tenía mucho para ordenar.

¿Qué pasó?

Llegaron algunos paquetes.

c. Contexto facilitador + cuantificador solo algunos (CFS)

El viernes envié unos regalos a mi familia para Navidad.

¿Llegaron a tiempo?

Llegaron solo algunos paquetes. El resto no llegó.

d. Contexto neutro + solo algunos (CNS)

El jueves en la oficina Pedro tenía mucho para ordenar.

¿Qué pasó?

Llegaron solo algunos paquetes. El resto no llegó.

e. Oraciones aceptables (EAC)

El viernes compré unos libros para mi familia.

¿Qué compraste?

A mis padres les compré dos novelas y a mi hermano un cómic. 


\section{f. Oraciones inaceptables (EIC)}

Mañana viene el electricista.

¿Qué día cae Navidad?

Comemos a las tres de la tarde. Yo voy a estar para cocinar.

Todas las condiciones estaban emparejadas en cantidad de cláusulas y la cantidad de palabras presentaba una variabilidad de $+/-3$, excepto en los casos de CNA donde, como ya mencionamos, contaban con una cláusula menos. Las listas fueron organizadas siguiendo un patrón de cuadrado latino para evitar que se repitieran en cada lista contextos o frases con cuantificadores. En el análisis se tuvieron en cuenta la misma cantidad de sujetos para cada lista $(n=65)$.

\subsubsection{Procedimiento}

La tarea consistía en indicar el grado de naturalidad de los estímulos, seleccionado un botón de una escala Likert con 7 puntos. Esta escala se conformó con dos extremos (poco natural y muy natural) y los puntos intermedios fueron etiquetados únicamente con números. Esto permite, como plantea Cowart (1997), utilizar métodos estadísticos para datos de intervalos. El diálogo se presentaba de manera completa, en tres líneas, tal como aparece en los ejemplos de (4). El contexto se encontraba marcado en itálica, mientras que el resto del diálogo se encontraba en letra normal y sin ninguna marca extra. Los botones de respuesta se encontraban debajo del texto. Entre los estímulos, se presentaba una pantalla de descanso que también cumplía la función de reajustar la posición del puntero del mouse, esto aseguraba que la distancia recorrida entre la posición inicial del puntero y el botón de respuesta fuera siempre la misma y se pudieran utilizar los tiempos de respuesta como datos válidos para el análisis. Los sujetos tenían tiempo límite de $13000 \mathrm{~ms}$ para realizar el juicio, transcurrido este tiempo se pasaba al siguiente estímulo y el anterior no podía ser ya evaluado.

Los estímulos fueron administrados vía web utilizando un código en .html y JavaScript con la librería JsPsych (De Leeuw, 2015). Dado que el experimento fue distribuido por internet, los participantes realizaron la actividad en sus computadoras personales y en diferentes ámbitos. Para garantizar la validez de los datos se tuvieron en cuenta distintas medidas de control sugeridas para ex- 
perimentos conducidos con esta metodología y que han sido validadas en diferentes oportunidades (Bartneck, Duenser, Moltchanova \& Zawieska, 2015; De Leeuw \& Motz, 2016; Enochson \& Culbertson, 2015; Sprouse, 2011). En primer lugar, la duración del experimento estaba limitaba a 15 minutos para garantizar que pudiera ser completada con las menores distracciones posibles. Al mismo tiempo, se les ofrecía a los participantes tiempo de descanso entre cada una de las respuestas, pudiendo interrumpir la actividad y retomarla sin perjudicar la validez de sus respuestas. En tercer lugar, se les solicitó a los participantes que registraran el tipo de mouse utilizado para luego comparar sus respuestas en función al tipo de equipo usado. Finalmente, se incluyeron en el diseño experimental estímulos especialmente diseñados para controlar y validar los datos obtenidos (condiciones EAC y EIC).

Como ya hemos mencionado, los estímulos estaban organizados en 4 listas que se les asignaban a los sujetos al azar y que seguían un esquema de cuadrado latino. Dentro de las listas, se crearon bloques que contenían un estímulo de cada condición seleccionado al azar. Por último, estos bloques se presentaban al participante siguiendo un orden aleatorio. De esta manera se aseguraba que ninguno de los 260 sujetos tuviera el mismo orden de presentación de los estímulos.

\subsubsection{Análisis de datos}

Los datos fueron analizados en R 3.3.2 y utilizamos el paquete \{lme4\} (Bates, Maechler, Bolker \& Walker, 2015) para realizar modelos lineales mixtos (linear mixed-effects models, LMM). Esta metodología presenta ventajas sobre el uso tradicional de múltiples ANOVAS, ya que permite que la varianza por sujeto y por estímulo sea considerada simultáneamente. De la misma manera, justificamos el uso de LMм para el análisis de escalas Likert siguiendo a Norman (2010). Los modelos fueron construidos siguiendo los lineamientos de Bates, Kliegl, Vasishth y Baayen (2015) con condición como efecto fijo y participante e ítem como efectos aleatorios. Finalmente, se utilizó el paquete \{multcomp (Hothorn, Bretz \& Westfall, 2008) para realizar comparaciones múltiples entre las condiciones y obtener los valores- $p$. En este caso, los contrastes fueron realizados con el método Tukey. Se incluye en el Anexo el código utilizado para este análisis, donde también se muestran los resultados de pruebas no paramétricas 
tradicionalmente utilizadas para este tipo de escalas y que coinciden con los resultados de los LMM, aunque muestran ser menos conservadoras (los valores- $p$ son más pequeños que los reportados por los LMM).

Para los datos de aceptabilidad solo se quitaron los casos donde no se llegó a dar una respuesta. En cuanto a los datos de tiempos de respuesta se seleccionaron solo aquellos participantes que realizaron la actividad en una computadora de escritorio con mouse ( $n=152$ ), manteniendo una misma cantidad de participantes por lista. Siguiendo este criterio, se eliminaron las respuestas menores a $2700 \mathrm{~ms}$ y mayores a $12500 \mathrm{~ms}$ que suponían un 4.24\% de los casos. A partir de los tiempos de respuesta y la cantidad de palabras de los estímulos, se calculó el ritmo de lectura, que resulta del cociente de ambos. Finalmente, se realizó una transformación del ritmo con el logaritmo natural.

\subsection{Resultados y discusión}

\subsubsection{Aceptabilidad}

En el caso de los estímulos de control, la media de aceptabilidad de los estímulos aceptables $($ EAC $\mathrm{mdn}=5.31)$ difiere de los inaceptables $($ EIC $\operatorname{mdn}=0.23)$ (coef $=-4.977, \mathrm{SE}=0.042, t=-116.6$ ). La prueba post-hoc de Tukey muestra que esta diferencia es significativa $(p>.0001)$. Estos resultados confirman la validez de los datos obtenidos mediante actividad en línea, ya que los participantes respondieron según lo esperado en los estímulos de control y mostraron una sensibilidad notable entre los dos extremos de aceptabilidad, lo que indica que efectivamente estaban cumpliendo con las instrucciones planteadas.

Para las condiciones con cuantificadores, como se puede ver en el Cuadro 1, las medias de puntaje de evaluación difieren mayormente para CNS en comparación con las otras condiciones.

Cuadro 1. Promedio de puntaje de evaluación por condiciones

\begin{tabular}{cccc}
\hline CNS & CNA & CFS & CFA \\
\hline $3.09(2.03)$ & $4.55(1.70)$ & $4.63(1.59)$ & $4.66(1.58)$ \\
\hline
\end{tabular}

Nota: La desviación estándar (DE) se presenta entre paréntesis. 
El análisis con LMM y la prueba post-hoc de Tukey muestran que esta diferencia es significativa, mientras que no hay diferencias entre las otras condiciones (cfr., Cuadro 2).

Cuadro 2. Análisis con modelos lineales mixtos y prueba post-hoc de Tukey para aceptabilidad

\begin{tabular}{lcccc}
\hline COMPARACIÓN & ESTIMACIÓN & ERROR ESTÁNDAR & $z$ & $p$ \\
\hline CNS X CNA & -1.451 & 0.0625 & -23.208 & $<.0001$ \\
CFA X CNS & 1.565 & 0.0628 & 24.907 & $<.0001$ \\
CFS X CNS & 1.528 & 0.0628 & 24.310 & $<.0001$ \\
CFA X CNA & 0.113 & 0.0619 & 1.839 & 0.255 \\
CFS X CNA & 0.076 & 0.0618 & 1.244 & 0.599 \\
CFS X CFA & -0.036 & 0.0622 & -0.594 & 0.934 \\
\hline
\end{tabular}

La diferencia en evaluación entre CNS y el resto de las condiciones nos muestra que el contexto afecta la evaluación de los estímulos cuando hay incongruencias entre el contexto y el cuantificador utilizado. En el caso de CNS el contexto no crea una referencia cuantificable a la cual el operador solo algunos pueda hacer referencia y por lo tanto hay una incongruencia que se refleja en un menor nivel de aceptabilidad. Es decir, el sujeto evalúa negativamente la relación entre el contexto neutro y el cuantificador solo algunos, ya que este operador hace referencia a un grupo que el contexto nunca habilitó ni planteó como relevante para la situación comunicativa. En cambio, en el caso de CFS, el contexto sí habilita una referencia cuantificable para el operador y por lo tanto la aceptabilidad es mayor. De la misma manera, en CNA y CFA los valores de aceptabilidad muestran que en ambos casos los contextos son compatibles con las dos interpretaciones posibles del cuantificador algunos. En el primer caso, no hay una referencia y el cuantificador se interpreta de manera lógica como un indefinido (existe al menos un $\mathrm{x}$ ). En el segundo caso, hay una referencia y el cuantificador se interpreta de manera enriquecida (algunos de los miembros de $\mathrm{x}$ y no todos).

Dado que los trabajos previos expuestos en el apartado anterior no presentaban juicios de aceptabilidad que indagaran sobre la influencia del contexto en el procesamiento de implicaturas, es posible argumentar que los resultados contrapuestos que obtienen estos experimentos pueden deberse a que otros factores, como el tipo de implicatura o la estructura de estilo indirecto, influyen en mayor 
medida en los datos obtenidos y que la influencia del contexto no sea realmente significativa.

\subsubsection{Tiempos de reacción}

En el Cuadro 3 presentamos la media del ritmo de lectura sin transformación logarítmica.

CuAdro 3. Media de ritmo por condición

\begin{tabular}{cccc}
\hline CNS & CNA & CFS & CFA \\
\hline $298.05(92.48)$ & $358.63(128.86)$ & $293.01(95.51)$ & 299.58 (95.30) \\
\hline Nota: La desviación estándar (DE) se presenta entre paréntesis. & &
\end{tabular}

Como se puede observar, la condición CNA presenta una mayor latencia que el resto de las condiciones, que parecen no diferir significativamente entre sí. El análisis con LMM y la prueba post-hoc de Tukey que se observa en el Cuadro 4 muestran que CNA difiere significativamente de las otras condiciones.

CuAdro 4. Análisis con modelos lineales mixtos y prueba post-hoc de Tukey para log10(ritmo)

\begin{tabular}{lcccc}
\hline COMPARACIÓN & ESTIMACIÓN & ERROR ESTÁNDAR & $z$ & $p$ \\
\hline CNS X CNA & -0.073 & 0.0060 & -12.18 & $>.0001$ \\
CFA X CNA & -0.072 & 0.0059 & -12.10 & $>.0001$ \\
CFS X CNA & -0.081 & 0.0060 & -13.55 & $>.0001$ \\
CFS X CNS & -0.001 & 0.0060 & 0.192 & 0.997 \\
CFS X CNS & -0.007 & 0.0060 & -1.306 & 0.559 \\
CFS X CFA & -0.009 & 0.0060 & -1.509 & 0.432 \\
\hline
\end{tabular}

El ritmo para esta tarea muestra que para CNA hay una mayor latencia que para el resto de las condiciones y que esta diferencia es significativa. En este sentido, los resultados son compatibles con las predicciones del modelo por default. En primer lugar, la diferencia entre CNA y CFA se explica porque para el caso de CNA es necesario integrar el contexto y cancelar la implicatura para arribar al significado de la oración con cuantificador. Este proceso demora el tiempo de respuesta, ya que le toma más tiempo a los sujetos generar el significado del estímulo 
que deben evaluar. Lo mismo sucede entre CNA y CNS o CFS, ya que en el primer caso es necesario generar y cancelar una implicatura mientras que en el segundo caso no hay implicatura, sino que se recurre al significado lógico-proposicional explícito que codifica el cuantificador solo algunos. Teniendo en cuenta lo expuesto sobre las tareas de juicios de aceptabilidad, esta latencia se generaría debido al acceso de información implícita en el momento de lectura del estímulo y no se produciría en el proceso posterior de evaluación, por lo que es posible decir que los resultados apoyan las hipótesis del modelo de procesamiento por default. Esta hipótesis se refuerza debido a que no hay diferencias significativas en el nivel de aceptabilidad otorgado para las dos condiciones (CNA y CFA).

\section{Conclusiones}

En este trabajo reportamos un experimento de juicios de aceptabilidad realizado con hablantes nativos de español. Nuestros resultados dieron cuenta de que el contexto puede influir en el procesamiento del significado del cuantificador algunos al facilitar o inhibir la interpretación pragmática del mismo. En otras palabras, muestran que el cuantificador puede tener diferentes interpretaciones según el contexto que lo precede. Asimismo, los tiempos de reacción se explican mejor por las predicciones del modelo de procesamiento por default o ranked-parallel. No obstante, consideramos que es necesario profundizar el análisis de este fenómeno con nuevos diseños experimentales que permitan analizar con mayor especificidad los tiempos de lectura de cada segmento de los estímulos. La lectura global del estímulo, que abarca el contexto, la frase con cuantificador y la frase con el resto se corresponden con las predicciones del modelo por default, pero es necesario dar cuenta de si la latencia en la lectura se produce en el segmento con el cuantificador y no es producto de un mayor costo de procesamiento de los contextos. Finalmente, también hay que resaltar el hecho de que el modelo literal-first no permite realizar una distinción entre implicaturas generalizadas y particularizadas, mientras que el modelo de procesamiento por default sí. Esta distinción central, que dio lugar a estas propuestas teóricas y experimentales, no ha sido abordada en profundidad y consideramos que puede brindar mayor evidencia sobre los mecanismos que subyacen al procesamien- 
to de todos los tipos de implicaturas. Queda por lo tanto abierto el interrogante para ser abordado en futuros trabajos.

\section{Referencias}

Asociación de Academias de la Lengua Española \& Real Academia Española (2009). Nueva gramática de la lengua española. Madrid: Espasa Libros.

Bartneck, Christoph; Duenser, Andreas; Moltchanova, Elena, \& Zawieska, Karolina (2015). Comparing the similarity of responses received from studies in Amazon's Mechanical Turk to studies conducted online and with direct recruitment. PLOS ONE, 10(4), 1-23. doi: 10.1371/journal.pone.0121595

Bates, Douglas; Kliegl, Reinhold; Vasishth, Shravan, \& BaAyen, R. Harald (2015). Parsimonious mixed models. arXiv e-prints. Recuperado de https://arxiv.org/pdf/1506.04967v2.pdf

Bates, Douglas; Maechler, Martin; Bolker, Ben, \& Walker, Steven (2015). Fitting Linear Mixed-Effects Models Using lme4. Journal of Statistical Software, 67(1), 1-48. doi:10.18637/ jss.v067.i01

Bergen, Leon, \& Gordner, Daniel (2012). Speaker knowledge influences the comprehension of pragmatic inferences. Journal of Experimental Psychology: Learning, Memory, and Cognition, 38(5), 1450-1460. doi: 10.1037/a0027850

Bezuidenhout, Anne, \& Cutting, J. Cooper (2002). Literal meaning, minimal propositions, and pragmatic processing. Journal of Pragmatics, 34(4), 433-456.

Breheny, Richard; Katsos, Napoleon, \& Williams, John (2006). Are generalized scalar implicatures generated by default? An on-line investigation into the role of context in generating pragmatic inferences. Cognition, 100(3), 434-463.

COWART, WAYNE (1997). Experimental syntax: Applying objective methods to sentence judgments. Mil Robles: Sage.

De Leeuw, Joshua (2015). jsPsych: A JavaScript library for creating behavioral experiments in a Web browser. Behavior Research Methods, 47(1), 1-12. doi: 10.3758/s13428-014-0458-y

De Leeuw, Joshua, \& Motz, Benjamin (2016). Psychophysics in a Web browser? Comparing response times collected with JavaScript and Psychophysics Toolbox in a visual search task. Behavior Research Methods, 48(1), 1-12. doi: 10.3758/s13428-015-0567-2

De Neys, Wim, \& Schaeken, Walter (2007). When people are more logical under cognitive load: Dual task impact on scalar implicature. Experimental Psychology, 54(2), 128-133. doi: 10.1027/1618-3169.54.2.128 
Dienes, Zoltán, \& Scott, Ryan (2005). Measuring unconscious knowledge: Distinguishing structural knowledge and judgment knowledge. Psychological Research, 69(5-6), 338-351.

Enochson, Kelly, \& Culbertson, Jennifer (2015). Collecting psycholinguistic response time data using Amazon Mechanical Turk. PLoS ONE, 10(3), e0116946. doi: 10.1371/journal. pone. 0116946

Grice, Herbert (1989). Studies in the way of words. Cambridge: Harvard University Press.

Horn, LAURENCE (1984). Toward a new taxonomy for pragmatic inference: Q-based and R-based implicatures. En Deborah Schiffrin (Ed.), Meaning, form, and use in context: Linguistic applications (pp. 11-42). Washington: Georgetown University Press.

Hothorn, Torsten; Bretz, Frank, \& Westfall, Peter (2008). Simultaneous inference in general parametric models. Biometrical Journal, 50(3), 346-363. doi: 10.1002/bimj.200810425

HuANG, Yi TING, \& SNEDEKER, JESSE (2009). Online interpretation of scalar quantifiers: Insight into the semantics-pragmatics interface. Cognitive Psychology, 58(3), 376-415. doi: 10.1016/j. cogpsych.2008.09.001

Huddleston, Rodney, \& Pullum, Geoffrey (Eds.) (2002). The Cambridge grammar of the English language. Cambridge: Cambridge University Press.

KATSOS, NAPOLEON (2009). Neither default nor particularised: Scalar implicature from a developmental perspective. En Uli Sauerland \& Kazuko Yatsushiro (Eds.), Semantics and pragmatics: From experiment to theory (pp. 51-73). Basingstoke: Palgrave Macmillan.

LAUTER, MirIAM (2013). If SOME folks are wise, are others otherwise? The interaction of context and emphasis in online scalar implicature processing (Tesis de licenciatura inédita). Yale University, New Haven.

Levinson, Stephen (1983). Pragmatics. Cambridge: Cambridge University Press.

Levinson, Stephen (2000). Presumptive meanings: The Theory of Generalized Conversational Implicature. Cambridge: MIT Press.

Lewis, Shevaun (2013). Pragmatic enrichment in language processing and development (Tesis doctoral inédita). University of Maryland, College Park.

Norman, Geoff (2010). Likert scales, levels of measurement and the "laws" of statistics. Advances in Health Sciences Education, 15(5), 625-632. doi: 10.1007/s10459-010-9222-y

Noveck, Ira, \& Reboul, Anne (2008). Experimental pragmatics: A Gricean turn in the study of language. Trends in Cognitive Sciences, 12(11), 425-431. doi: 10.1016/j.tics.2008.07.009

Politzer Ahles, Stephen; Fiorentino, Robert; Jiang, Xiaoming, \& Zhou, Xiaolin (2013). Distinct neural correlates for pragmatic and semantic meaning processing: An event-related 
potential investigation of scalar implicature processing using picture-sentence verification. Brain Research, 1490, 134-52.

Politzer-Ahles, Stephen, \& Husband, E. Matthew (2018). Eye movement evidence for context-sensitive derivation of scalar inferences. Collabra: Psychology, 4(1), 1-13. doi: 10.1525/ collabra. 100

Searle, John (1975). Indirect speech acts. En Peter Cole \& Jerry Morgan (Eds.), Syntax and semantics: Speech acts (Vol. 3, pp. 59-82). Nueva York: Academic Press.

Sperber, Dan, \& Wilson, Deirdre (1995). Relevance: Communication and cognition. 2a. ed. Oxford: Blackwell.

Sperber, Dan, \& Wilson, DeIrdre (2002). Pragmatics, modularity and mind-reading. Mind \& Language, 17(1-2), 3-23.

Sprouse, Jon (2011). A validation of Amazon Mechanical Turk for the collection of acceptability judgments in linguistic theory. Behavior Research Methods, 43(1), 155-167. doi: 10.3758/ s13428-010-0039-7

Topolinski, SAscha, \& Strack, Fritz (2009). The architecture of intuition: Fluency and affect determine intuitive judgments of semantic and visual coherence and judgments of grammaticality in artificial grammar learning. Journal of Experimental Psychology: General, 138(1), 39-63. doi: 10.1037/a0014678 


\section{Anexo: Bitácora del análisis estadístico}

En esta sección presentamos el procedimiento de análisis estadístico del experimento junto con el código utilizado.

5.1. Carga de datos y librerías a utilizar

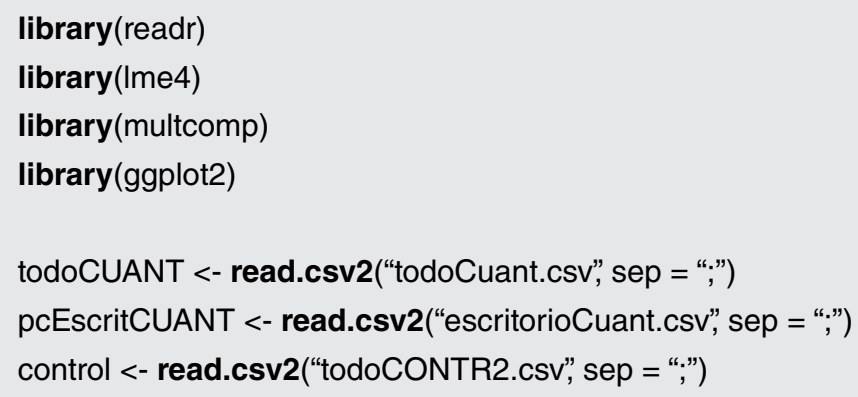

5.2. Análisis de los estímulos de control

Se corroboran las primeras líneas de los datos.

\begin{tabular}{|c|c|c|c|c|c|c|c|}
\hline \#\# & & sujeto & $\mathrm{rt}$ & index & aceptabilidad & item & condicion \\
\hline \#\# & 1 & 1 & 6725 & 16 & 2 & 1 & EAC \\
\hline \#\# & 2 & 1 & 3843 & 32 & 5 & 3 & EAC \\
\hline \#\# & 3 & 1 & 6348 & 62 & 6 & 5 & EAC \\
\hline \#\# & 4 & 1 & 6419 & 68 & 5 & 2 & EAC \\
\hline \#\# & 5 & 1 & 10402 & 88 & 6 & 4 & EAC \\
\hline \#\# & 6 & 2 & 12299 & 20 & 6 & 1 & EAC \\
\hline
\end{tabular}

Se realiza un histograma a fin de analizar la distribución de las respuestas para los estímulos de control. El histograma se muestra en la Figura 1. 

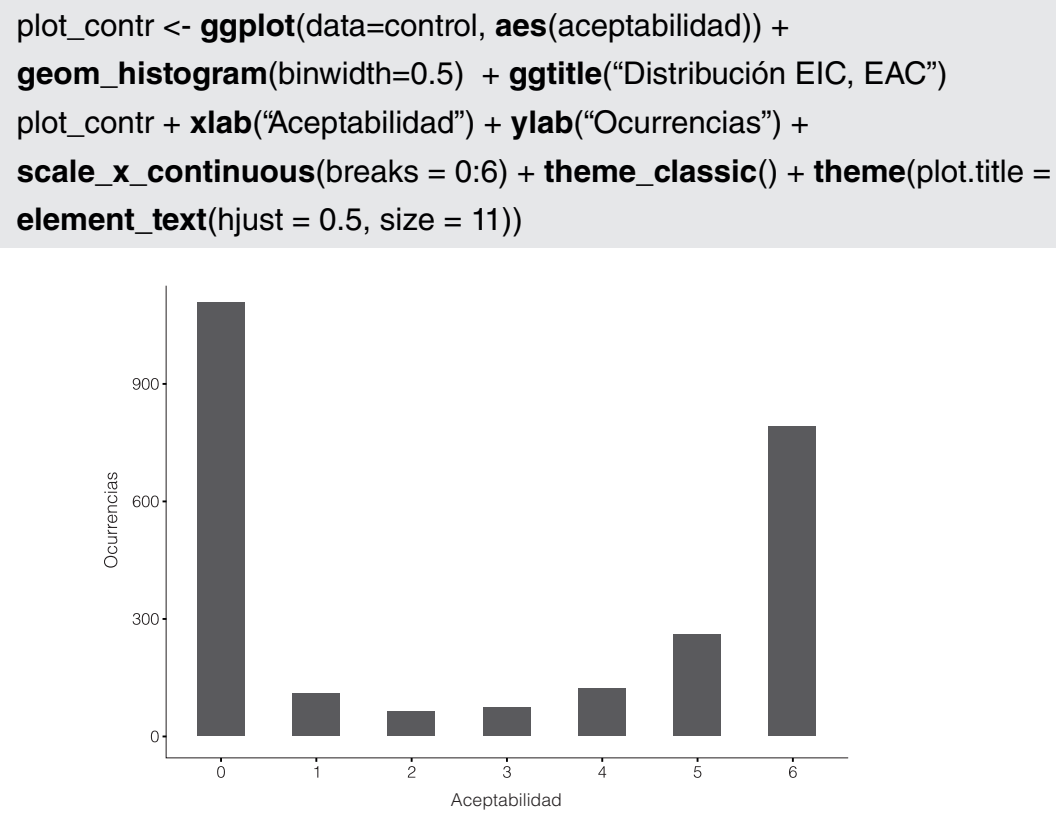

FIGURA 1. Distribución de aceptabilidad EIC, EAC

Presenta una distribución característica de escalas Likert. Los LMM han mostrado proveer una baja tasa de errores de tipo I en distribuciones no normales. De la misma manera, siguiendo a Norman (2010), se justifica utilizar descripciones paramétricas de los datos, ya que se utiliza una escala de 7 puntos y solo se etiquetan los extremos. Se incluyen al final los resultados realizados con pruebas no paramétricas (Wilcoxon), que han arrojado los mismos niveles de significatividad.

Realización del resumen de los datos. Se indica la media y la desviación estándar.

$$
\begin{aligned}
& \text { EAC <- control\$aceptabilidad[control\$condicion == 'EAC'] } \\
& \text { EIC <- control\$aceptabilidad[control\$condicion == 'EIC'] } \\
& \text { meanControl }<- \text { c }(\text { mean }(E A C), \text { mean }(E I C)) \\
& \text { sdControl <- c }(\text { sd(EAC), sd(EIC)) } \\
& \text { filControl } \quad<- \text { c('EAC', 'EIC') }
\end{aligned}
$$




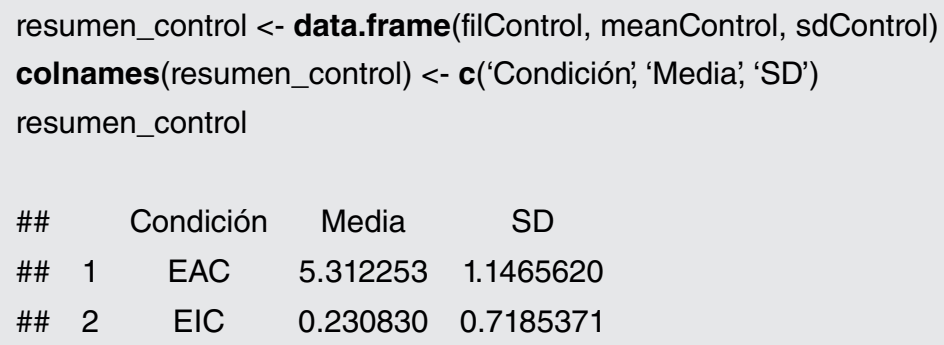

Creamos un modelo lineal de efectos mixtos (LMM) con condición (EAC, EIC) como efecto fijo y sujeto e ítem como efectos random. Luego utilizamos la función glht del paquete multcomp para hacer comparaciones múltiples entre las condiciones utilizando el método de Tukey.

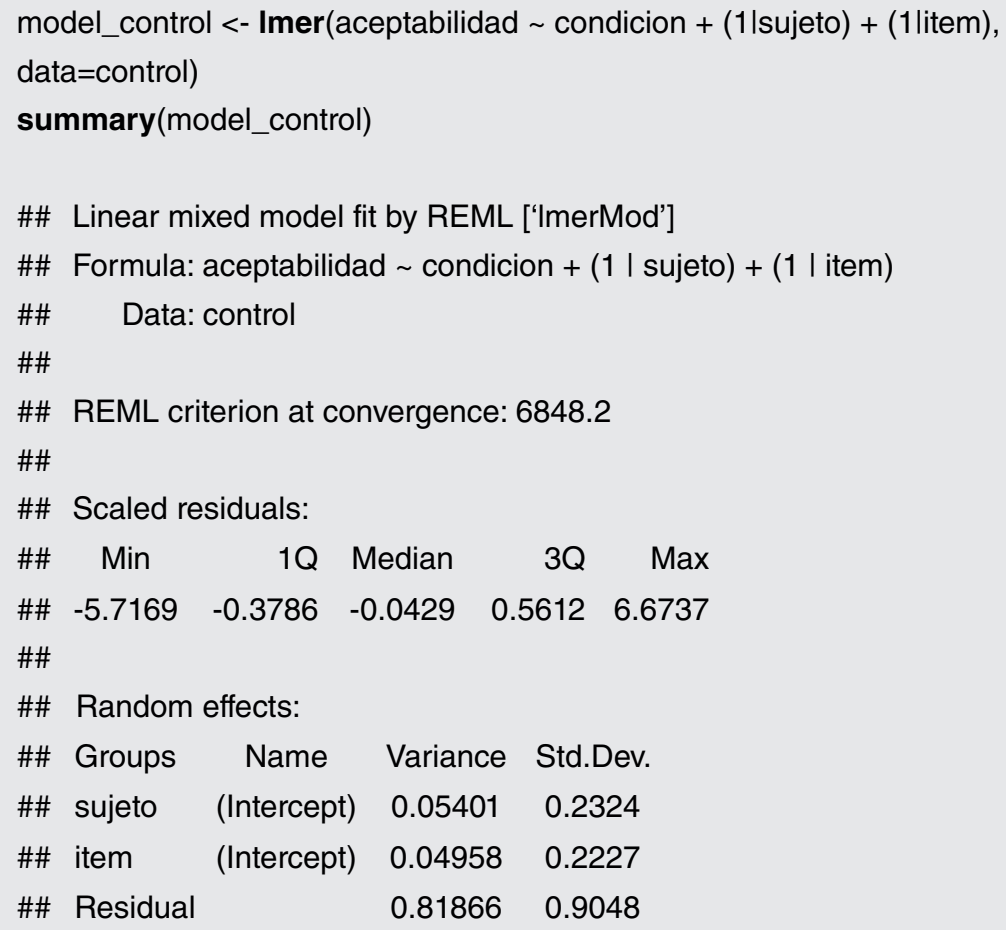




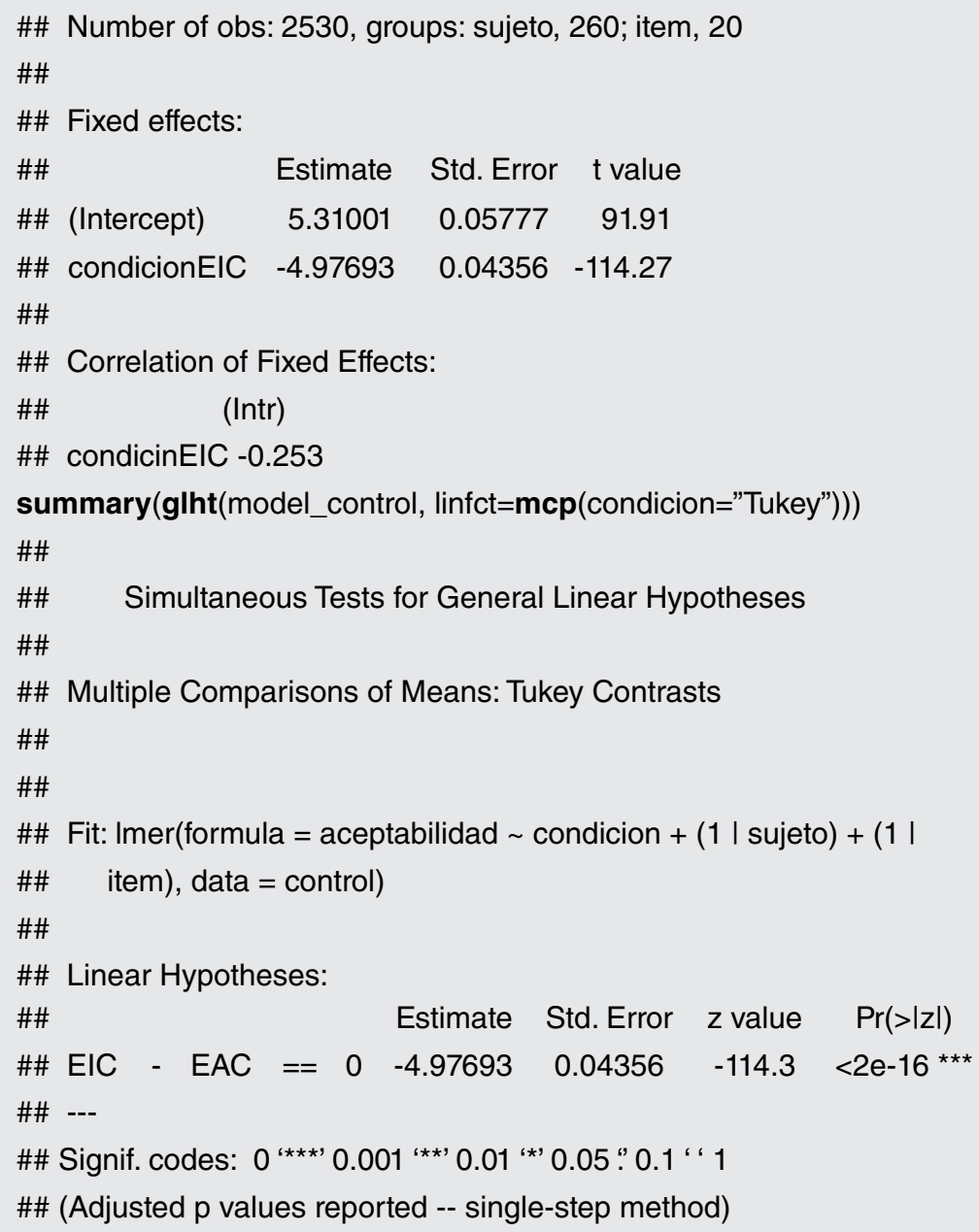

El análisis muestra que las diferencias entre EIC y EAC son significativas.

\subsection{Análisis de aceptabilidad}

Se corroboran las primeras líneas de los datos. 


\begin{tabular}{lccccccc}
\multicolumn{7}{l}{ head(todoCUANT $[\mathbf{c}(1,2,3,5,6,7)])$} \\
$\# \#$ & sujeto & rt & index & aceptabilidad & condicion & item \\
$\# \#$ & 1 & 1 & 3555 & 22 & 6 & CNS & 2 \\
$\# \#$ & 2 & 1 & 4047 & 24 & 3 & CFA & 5 \\
$\# \#$ & 3 & 1 & 2157 & 26 & 6 & CNA & 15 \\
$\# \#$ & 4 & 1 & 8470 & 30 & 5 & CFS & 11 \\
$\# \#$ & 5 & 1 & 7284 & 34 & 2 & CFA & 3 \\
$\# \#$ & t6 & 1 & 9105 & 36 & 1 & CNS & 3
\end{tabular}

Se realiza un histograma para analizar la distribución de las respuestas para los estímulos blanco. El histograma se muestra en la Figura 2.
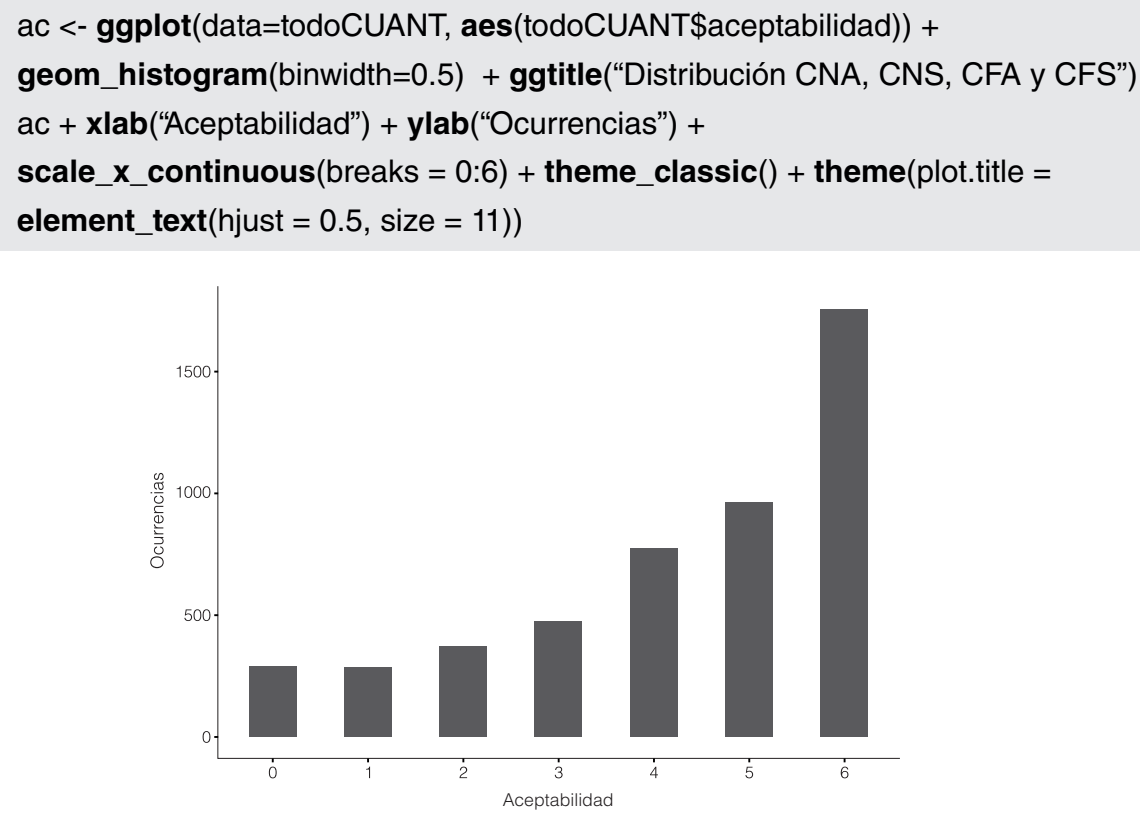

FIGURA 2. Distribución de aceptabilidad CNA, CNS, CFA y CFS

Realización del resumen de los datos. Se indica la media y la desviación estándar. 


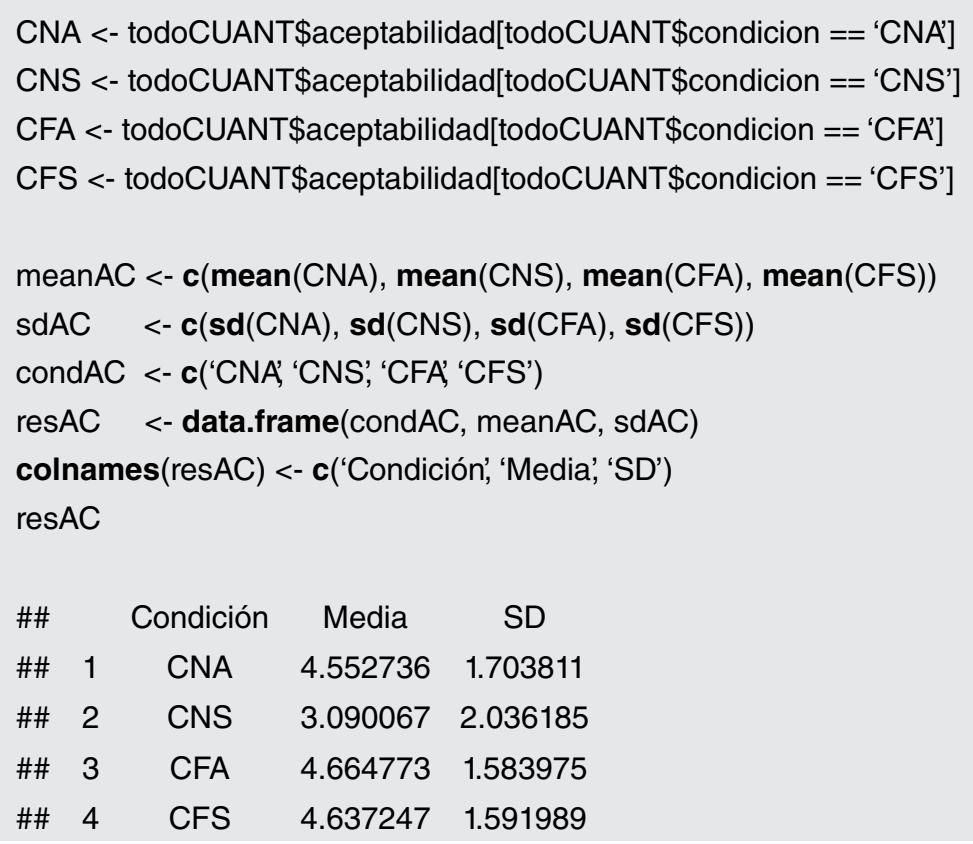

$\mathrm{Al}$ igual que el caso anterior, presenta una distribución característica de las escalas Likert. Justificamos el análisis de la misma manera.

Creamos un modelo lineal de efectos mixtos (LMM) con condición como efecto fijo y sujeto e ítem como efectos random. Luego realizamos las comparaciones múltiples utilizando la función glht del paquete multcomp.

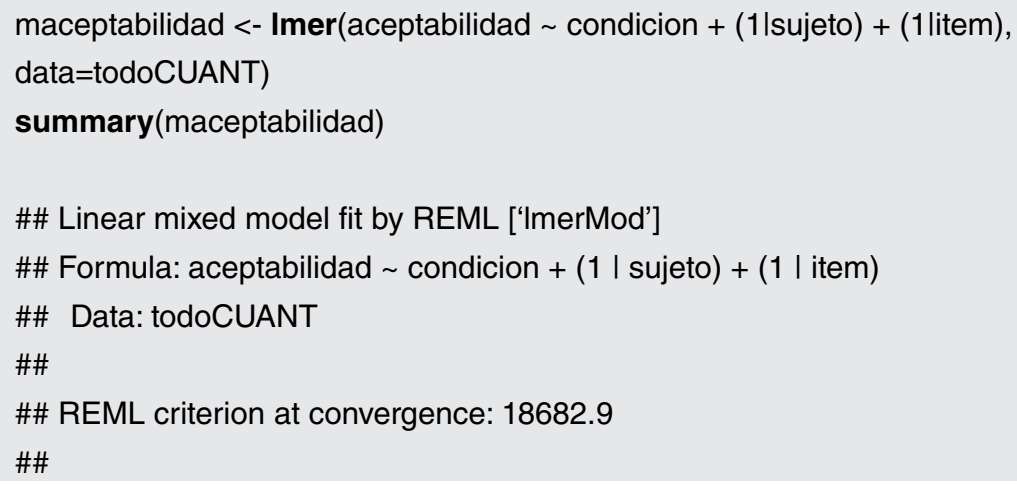




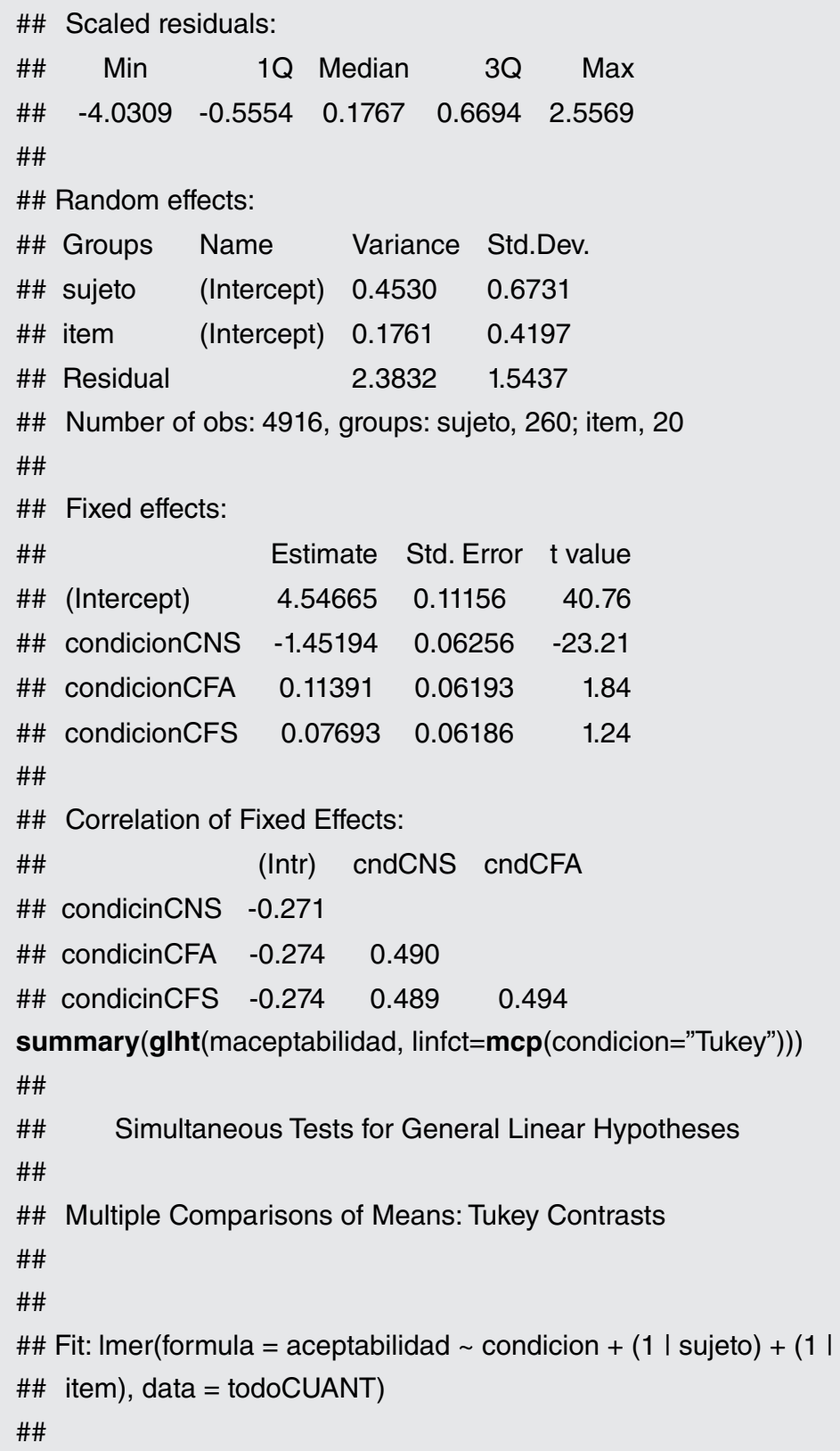




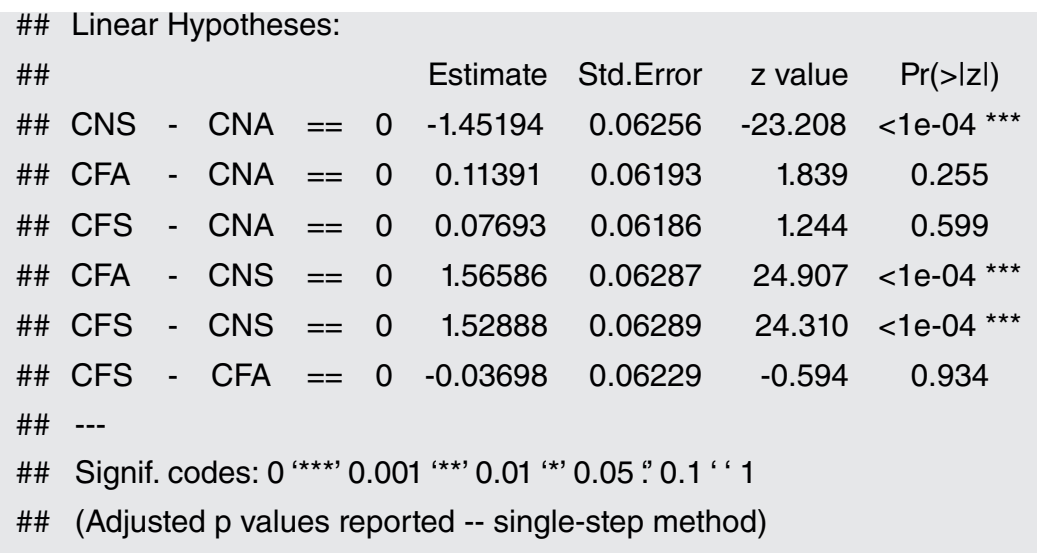

El análisis muestra que las diferencias entre CNS y el resto de las condiciones son significativas, mientras que las diferencias entre CNA, CFS y CFA no lo son.

\subsection{Análisis tiempo de respuesta}

Se corroboran las primeras líneas de los datos.

head(pcEscritCUANT $[\mathbf{c}(1,2,3,5,6,7,8,9,10)])$
$\begin{array}{lccccccccc}\# \# & \text { sujeto } & \text { rt } & \text { index } & \text { aceptabilidad } & \text { item } & \text { condicion } & \text { cantPal } & \text { ritmo } & \text { logRitmo } \\ \# \# 1 & 1 & 3555 & 22 & 6 & 2 & \text { CNS } & 20 & 177.7500 & 2.249810 \\ \# \# 2 & 1 & 4047 & 24 & 3 & 5 & \text { CFA } & 24 & 168.6250 & 2.226922 \\ \# \# 3 & 1 & 2157 & 26 & 6 & 15 & \text { CNA } & 20 & 107.8500 & 2.032820 \\ \# \# 4 & 1 & 8470 & 30 & 5 & 11 & \text { CFS } & 29 & 292.0690 & 2.465485 \\ \# \# 5 & 1 & 7284 & 34 & 2 & 3 & \text { CFA } & 38 & 191.6842 & 2.282586 \\ \# \# 6 & 1 & 9105 & 36 & 1 & 3 & \text { CNS } & 42 & 216.7857 & 2.336031\end{array}$

Se realiza un histograma para analizar la distribución de los tiempos de respuesta para los estímulos blanco. El histograma se muestra en la Figura 3.

$$
\begin{aligned}
& \text { ac <- ggplot(data=pcEscritCUANT, aes(pcEscritCUANT\$logRitmo)) + } \\
& \text { geom_histogram() + ggtitle(“Distribución CNA, CNS, CFA y CFS”) }
\end{aligned}
$$




$$
\begin{aligned}
& \text { ac }+\mathbf{x l a b}(\text { (log10(Ritmo)") }+ \text { ylab(“Ocurrencias”) }+ \text { theme_classic }()+ \\
& \text { theme(plot.title }=\text { element_text(hjust }=0.5, \text { size }=11))
\end{aligned}
$$

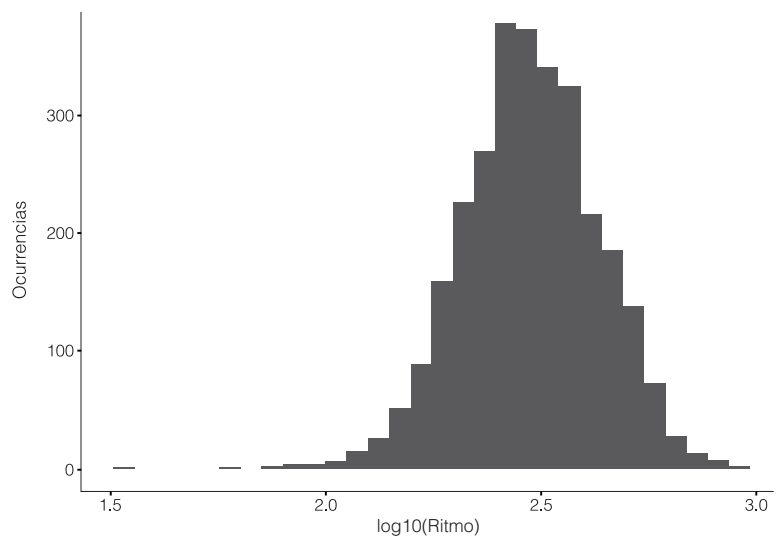

FigURA 3. Distribución log10(ritmo) CNA, CNS, CFA y CFS sin eliminación de outliers

La distribución es normal, pero se observan algunos outliers en la cola izquierda. Eliminamos estas ocurrencias e indicamos el porcentaje de ocurrencias eliminadas.

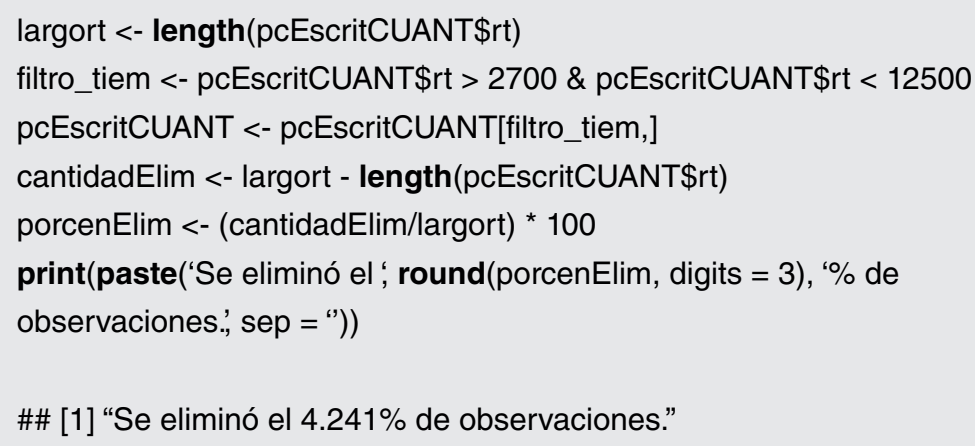

Se grafica nuevamente la distribución. El histograma se puede observar en la Figura 4.

$$
\begin{aligned}
& \text { ac <- ggplot(data=pcEscritCUANT, aes(pcEscritCUANT\$logRitmo)) + } \\
& \text { geom_histogram()+ ggtitle(“Distribución CNA, CNS, CFA y CFS”) }
\end{aligned}
$$



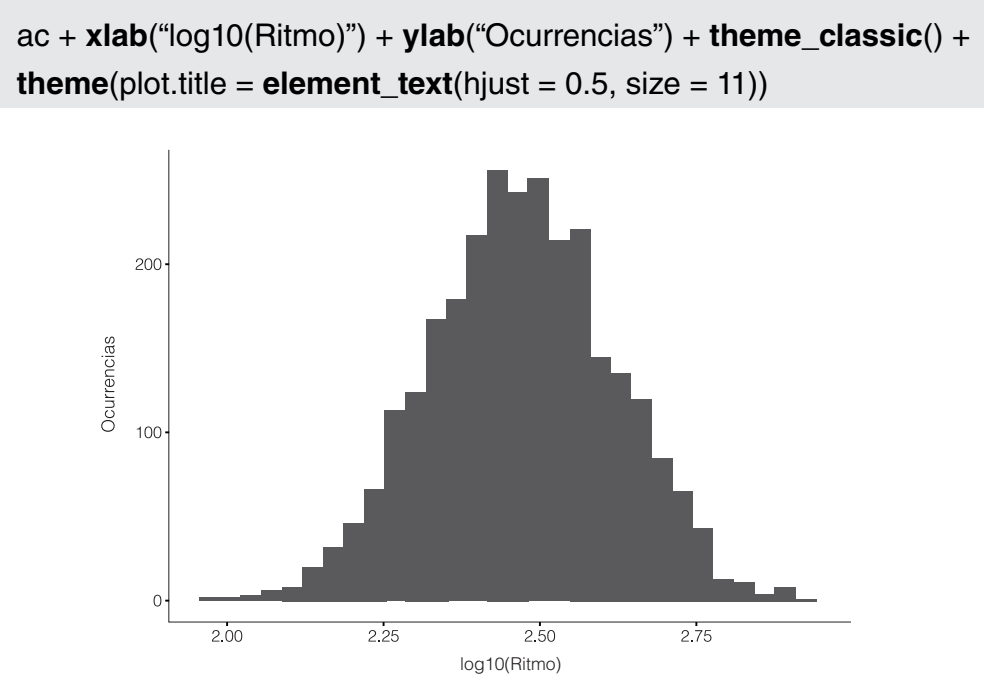

FIGURA 4. Distribución log10(ritmo) CNA, CNS, CFA y CFS con eliminación de outliers

Realización del resumen de los datos. Se indica la media y la desviación estándar.

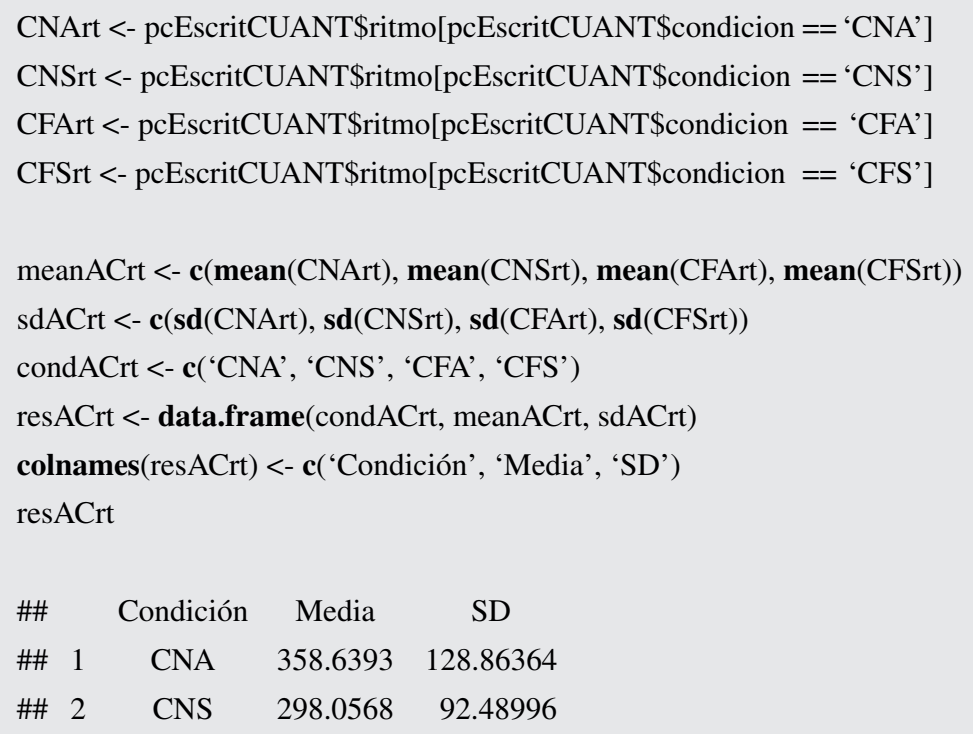




$\begin{array}{lllll}\text { \#\# } & 3 & \text { CFA } & 299.5882 & 95.30318 \\ \text { \#\# } & 4 & \text { CFS } & 293.0103 & 95.51081\end{array}$

Creamos un modelo lineal de efectos mixtos (LMM) con condición como efecto fijo y sujeto e ítem como efectos random. Luego realizamos las comparaciones múltiples utilizando la función glht del paquete multcomp.

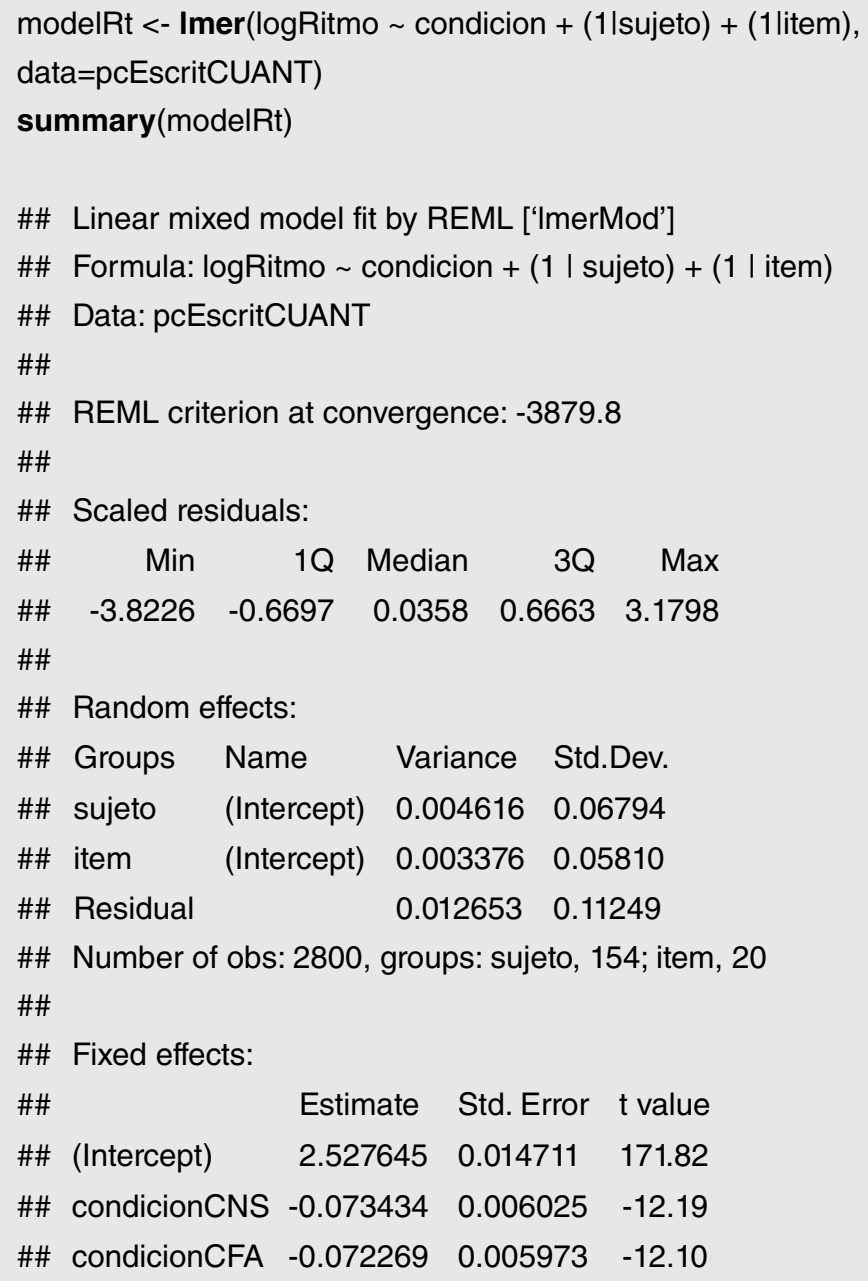




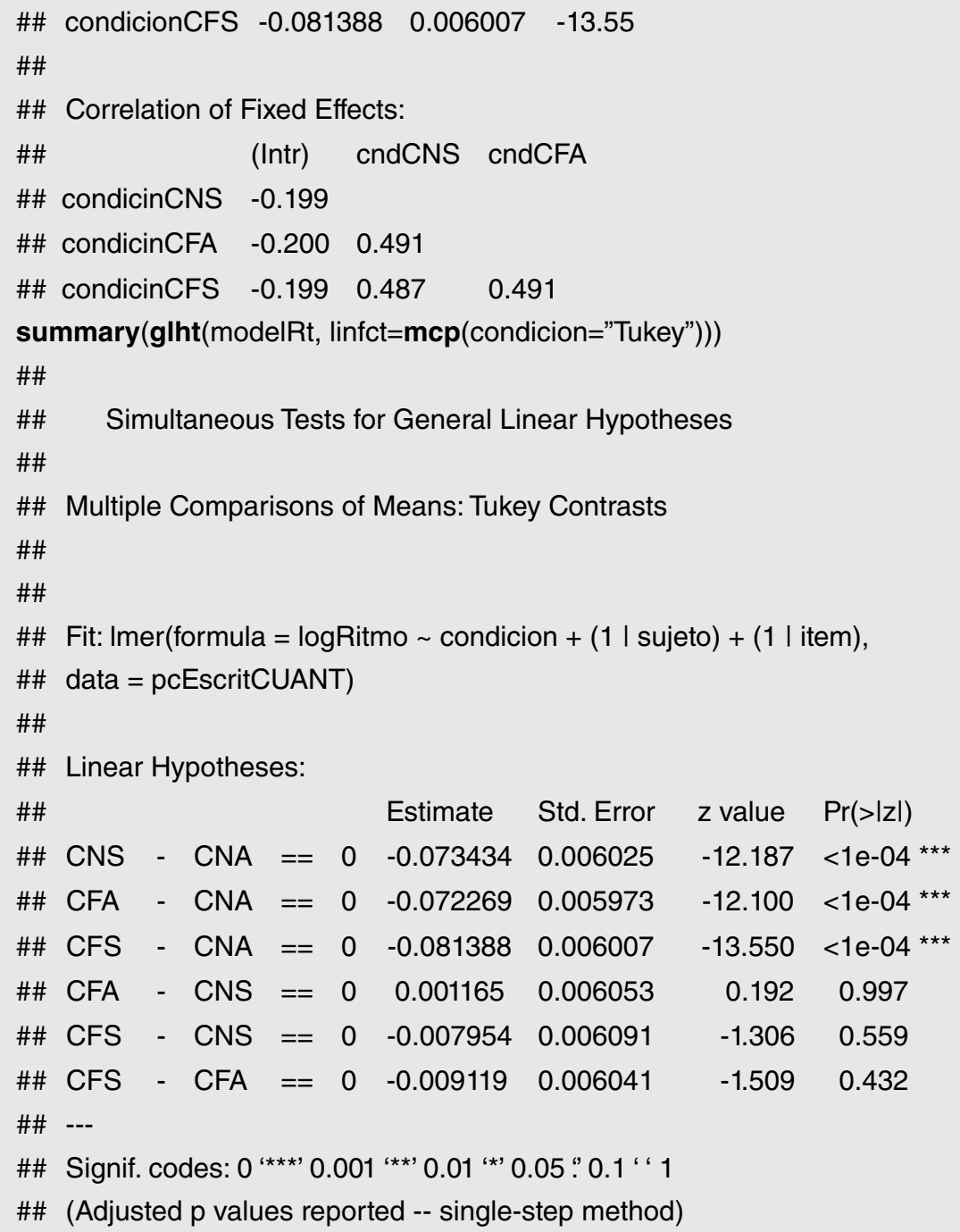

El ritmo para esta tarea muestra que para CNA hay una mayor latencia que para el resto de las condiciones y que esta diferencia es significativa. 


\subsection{Análisis con métodos no paramétricos}

Presentamos el análisis de los datos con modelos no paramétricos. En este caso, las respuestas de cada sujeto fueron promediadas por condición. De esta manera, cada sujeto aporta una observación por condición resultante del promedio de sus respuestas. Utilizamos la librería dyplr para realizar esta tarea.

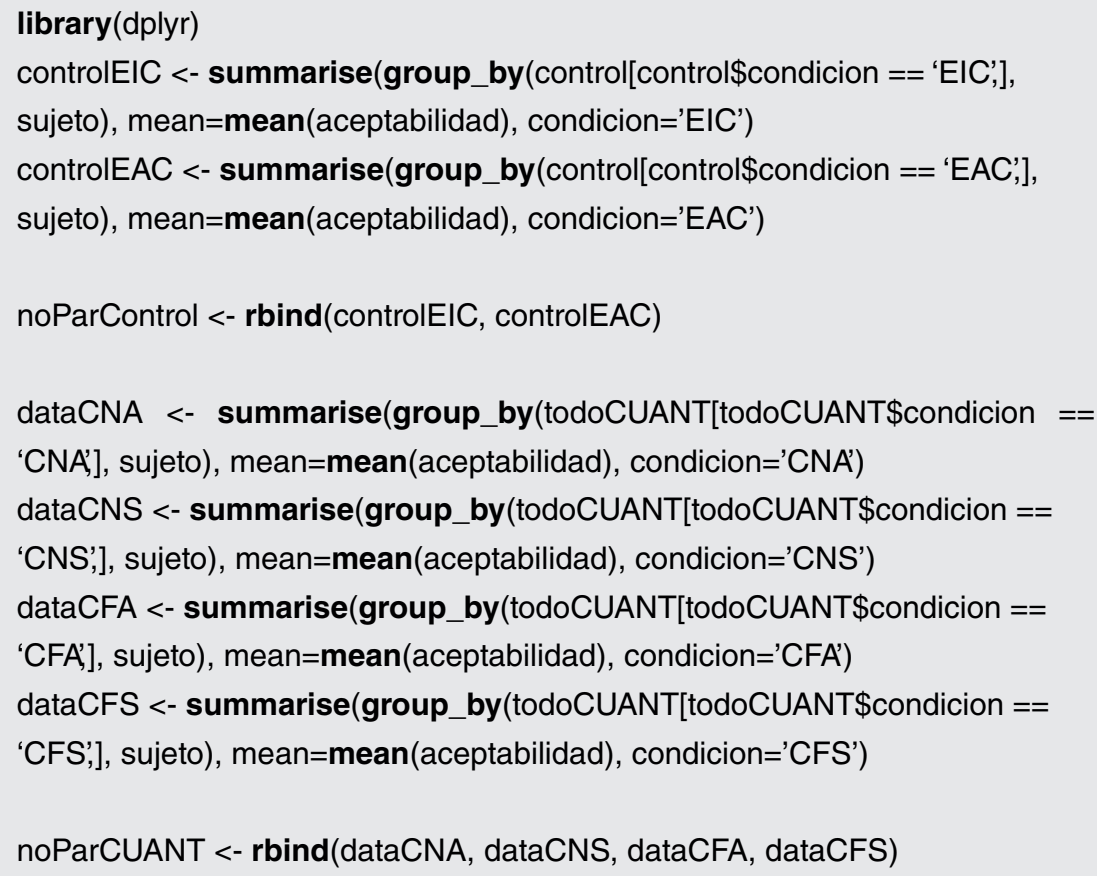

\subsubsection{Estímulos de control}

Se crean los vectores.

$$
\begin{aligned}
& \text { noParEAC <- noParControl\$mean[noParControl\$condicion == 'EAC'] } \\
& \text { noParEIC <- noParControl\$mean[noParControl\$condicion == 'EIC'] }
\end{aligned}
$$


Se realiza test de Wilcoxon.

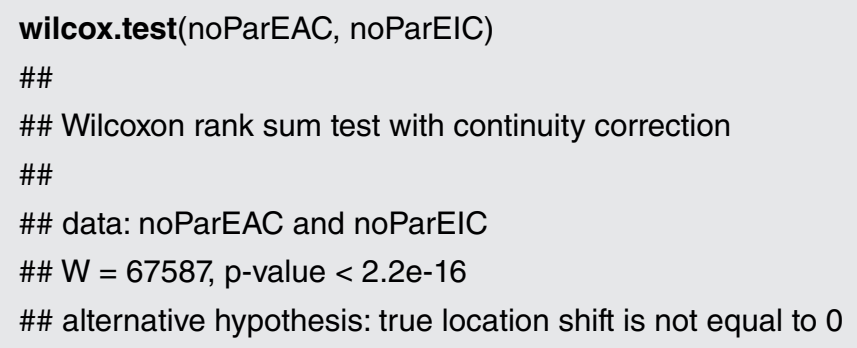

Como vemos, en este caso el análisis otorga un $p$-valor más pequeño que el realizado con los LMM.

\subsubsection{Estímulos con cuantificadores}

Se crean los vectores.

$$
\begin{aligned}
& \text { noParCNA <- noParCUANT\$mean[noParCUANT\$condicion == 'CNA'] } \\
& \text { noParCNS <- noParCUANT\$mean[noParCUANT\$condicion == 'CNS'] } \\
& \text { noParCFA <- noParCUANT\$mean[noParCUANT\$condicion == 'CFA'] } \\
& \text { noParCFS <- noParCUANT\$mean[noParCUANT\$condicion == 'CFS'] }
\end{aligned}
$$

Realización de test de Kruskal-Wallis para buscar diferencias entre las condiciones.

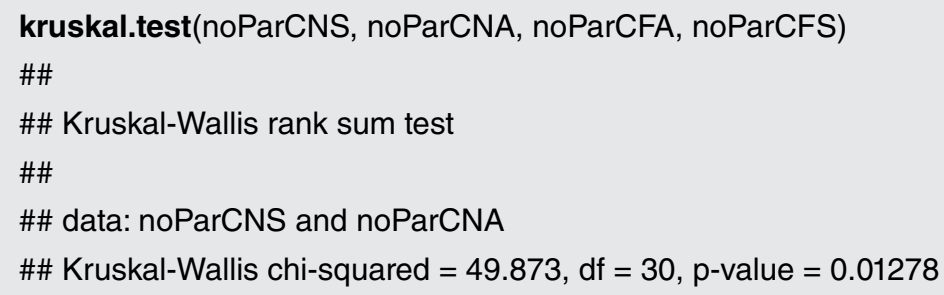

Este análisis nos muestra que existen diferencias entre las condiciones. Por lo tanto, procedemos a realizar comparaciones múltiples utilizando Wilcoxon. 


\begin{tabular}{|c|c|c|c|}
\hline & CNSxCN & $A<-$ wi & cox.test(noParCNS, noParCNA) \\
\hline & CFAxCN & $S<-$ wi & cox.test(noParCFA, noParCNS) \\
\hline & CFSxCN & $S<-$ wi & cox.test(noParCFS, noParCNS) \\
\hline & CFAxCN & $A<-$ wi & cox.test(noParCFS, noParCFA) \\
\hline & CFSxCN & $A<-$ wi & cox.test(noParCFS, noParCNA) \\
\hline & CFSxCF/ & $A<-$ wi & cox.test(noParCFS, noParCFA) \\
\hline & pvalores & $<-\mathrm{c}$ (CNS) & CNA\$p.value,CFAxCNS\$p.value, CFSxCNS\$p.value, \\
\hline & CFAxCN & A \$p.value & CFSxCNA\$p.value, CFSxCFA\$p.value) \\
\hline & $W<-c(C$ & NSxCNA $\$$ & statistic,CFAxCNS\$statistic, CFSxCNS\$statistic, \\
\hline & CFAxCN & A\$statistic & CFSxCNA\$statistic, CFSxCFA\$statistic) \\
\hline & compara & cion $<-\mathbf{c}(‘$ & ENSxCNA' 'CFAxCNS', 'CFSxCNS', 'CFAxCNA' 'CFSxCNA' \\
\hline & 'CFSxCF & & \\
\hline & reporte $<$ & - data.fra & ee(comparacion, W, format (pvalores, scientific = FALSE)) \\
\hline & colname & $\mathbf{s}$ (reporte) & <- c('Comparación', 'W', 'p-valor’) \\
\hline & reporte & & \\
\hline \#\# & Comparación & W & $\mathrm{p}$-valor \\
\hline \#\# 1 & CNSXCNA & 12287.0 & 0.0000000000000000000000000000000000032658785588 \\
\hline \#\# 2 & CFAxCNS & 56306.0 & 0.0000000000000000000000000000000000000018203163 \\
\hline \#\# 3 & CFSxCNS & 56406.5 & 0.0000000000000000000000000000000000000008283722 \\
\hline \#\# 4 & CFAXCNA & 33250.0 & 0.7479916170067033576174253539647907018661499023 \\
\hline \#\# 5 & CFSXCNA & 35287.0 & 0.3847630958378582821666213931166566908359527588 \\
\hline \#\# 6 & CFSxCFA & 33250.0 & 0.7479916170067033576174253539647907018661499023 \\
\hline
\end{tabular}

Como se puede observar, en este caso los resultados concuerdan con el análisis realizado con LMM y muestran un $p$-valor menos conservador. 